\title{
6. Electron Microscope Study of the Cardiac Conduction System
}

\author{
KeISHIRo KaWAMUra \\ [The Central Clinical Laboratory, Kyoto University Hospital, Kyoto]
}

KUNIKI HAYASHI

[The 3rd Division, Department of Internal Medicine, Kyoto University Faculty of Medicine, Kyoto]

In the hearts of rabbit, dog, cow, monkey and man, the ultrastructures of the S-A and A-V nodes are very similar to one another in each animal species and are definitely differentiated from those of PURKInJe fibers, and ordinary auricular, atrial and ventricular myocardium. The main portion of each node is characterized by a complicated disposition of small and irregularly shaped muscle cells with large nuclei and the presence of numerous nerve fibers in the interstitial space. Each nodal cell is enveloped by plasma membrane and closely connected with the adjacent cells by apposition of their membranes.
The plasma membranes bordering on the interstitial space are frequently associated with pinocytic vesicles and are covered by a basement membrane which extends from one cell surface to another. The plasma membrane at the cell junction is continuous with that which borders on the interstitium. Along the cell junction, several anatomically distinct regions may be identified as in approximately the same manner as in the intercalated disc of ordinary myocardial cells, i. e., the interfibrillar region of attachment of myofibrils, desmosomes, undifferentiated regions of closely apposed plasma membranes and nexuses. The gap between the

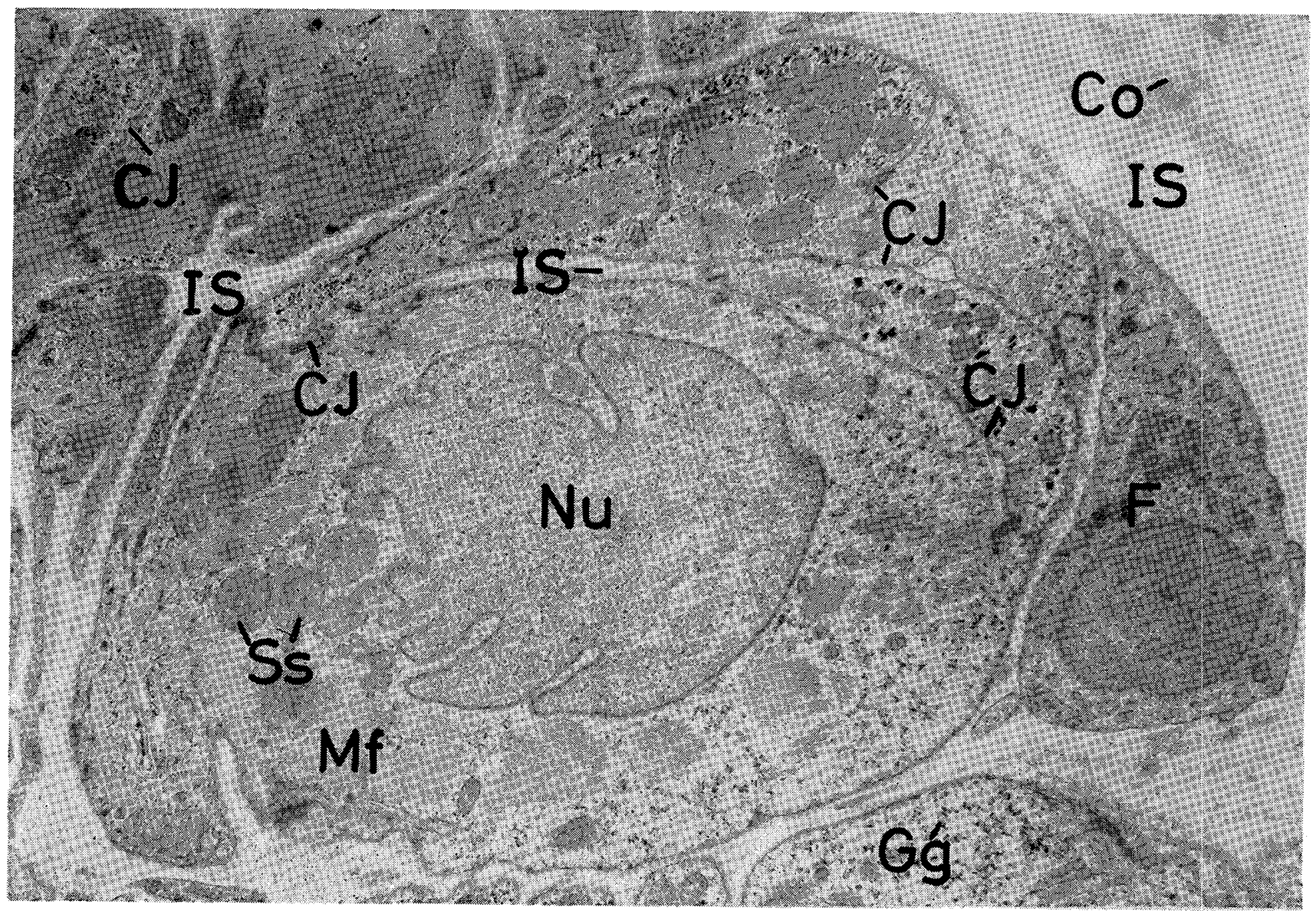

Fig. 1. S-A node of monkey heart $\times 6,200$

(Received for Publication, November 24, 1965)

Japanese Circulation Journal Vol. 3o, February 1966 
plasma membranes along the cell junction is about $200 \AA$ or more except for the nexal region. Although the details may have to wait for the observations following permanganate fixation, the structure of the nexus is rarely encountered in osmium preparations of the main portion of the S-A and A-V nodes.

The nervous elements found in nodal regions mostly consist of nonmyelinated nerve fibers. In general, the nerve fibers run separately or in a few groups, and some of the fibers are seen to be very near the surface of nodal muscle cells. Occasionally, the axons contain vesicular structures which are frequently identical in appearance with synaptic vesicles and are much less commonly similar to granular vesicle in nerve endings in other tissues. A few of the small mitochondria, neurofilaments and infrequent clusters of small dense granules are also seen in the axoplasm.

The Purkinje fibers of the cow, a represen- tative of the Artiodactyla, are markedly different in structure from those of other animal species so far investigated. In the cow, the Purkinje cells are huge and cylindrical or cuboidal; they are connected in several rows with each plasma membrane and form a core surrounded by the basement membrane. In the dog and rabbit, the Purkinje cells appear to be rather similar to ordinary myocardial cells in appearance and are connected to each other at the intercalated disc. The myofibrils are, however, occasionally few and may branch in various directions in the sarcoplasm; the sarcolemma may show some irregular outline and the sarcosomes appear smaller. In man and the monkey, the appearance of PukinJe cells in false tendons is in general quite similar to that of ordinary myocardial cells. They can hardly be differentiated from each other in many sections.

In these animals, however, there are some

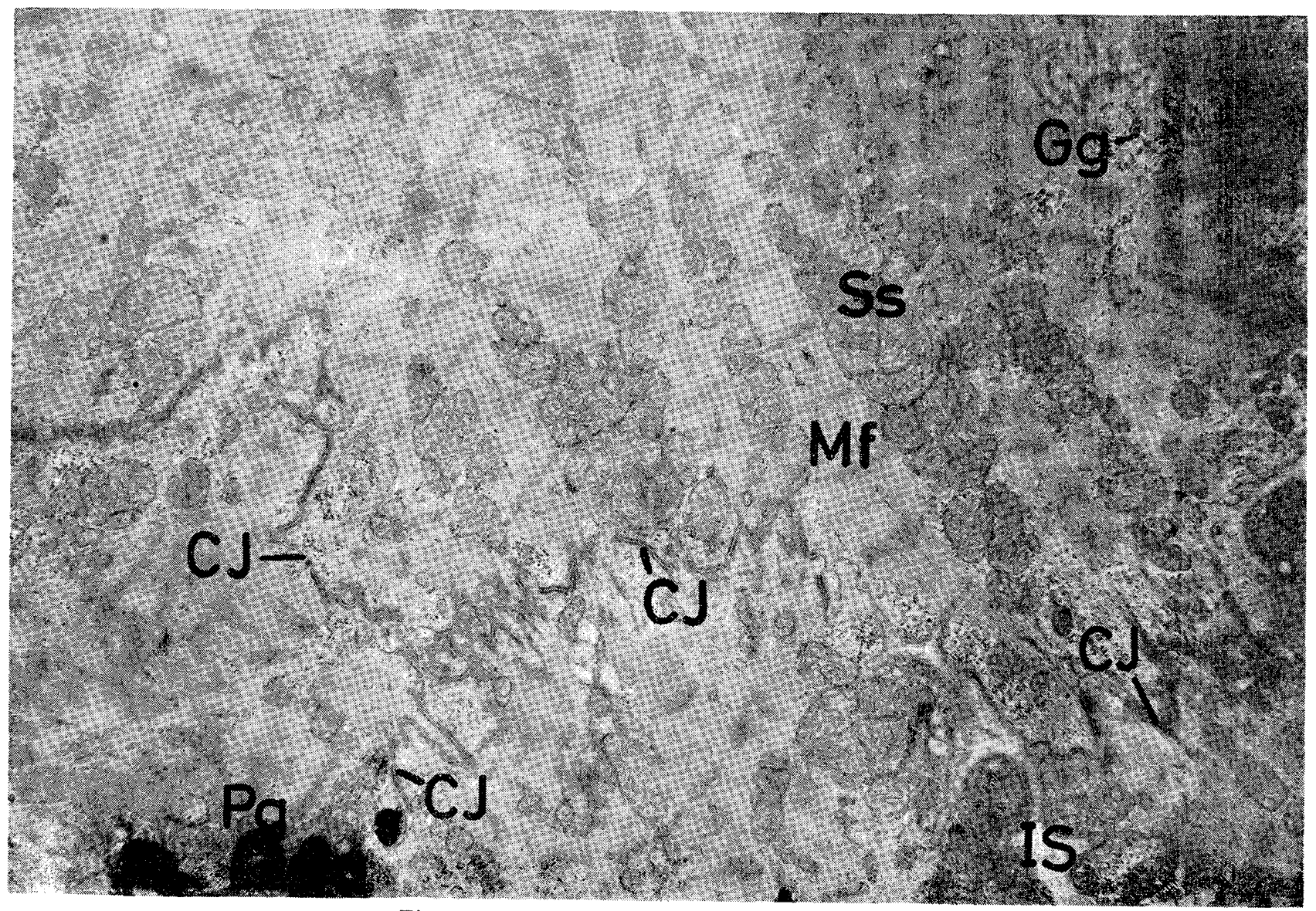

Fig. 2. Purkinje cells of human heart. $\times 6,000$

Abbreviations in Figs. 1 and 2.

$\mathrm{CJ}$, cell junction; $\mathrm{CO}$, collagen fibrils; F, fibroblast; $\mathrm{Gg}$, glycogen granules; IS, interstitial space ; Mf, myofibril; Nu, nucleus; Pg, pigment granules; Ss, sarcosomes. 
sites where three cell junctions meet one point in the micrographs of PURKinje cells. This finding gives evidence of peculiar cellular disposi- tion different from that of ordinary myocardial cells.

\section{Effect of Ischemia on the Atrio-ventricular Conduction System in the Dog ${ }^{\dagger}$ Kunitake Hashiba \\ [The First Department of Internal Medicine (Director: Prof. Y. Takaoka), Nagasaki University School of Medicine, Nagasaki]}

The purpose of this study is to investigate the effect of ischemia upon the $\mathrm{A}-\mathrm{V}$ conduction system. Only a few reports have been published on this subject ${ }^{1-4)}$.

(b)

(a)

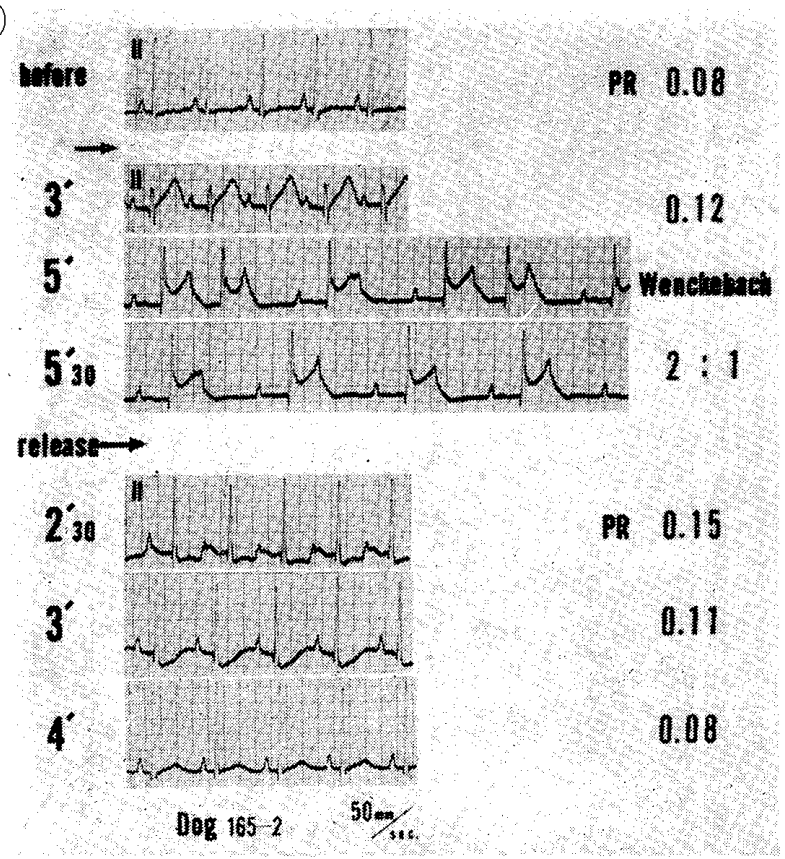

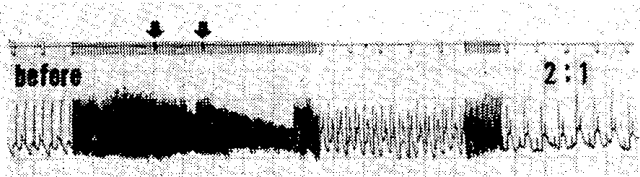

3

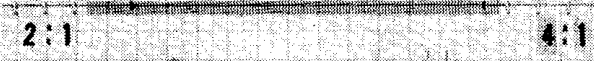

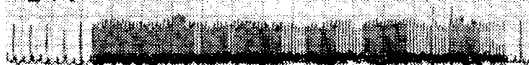

Wi.

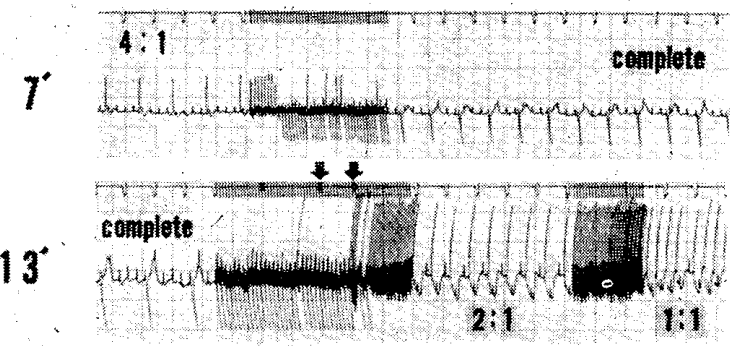

14

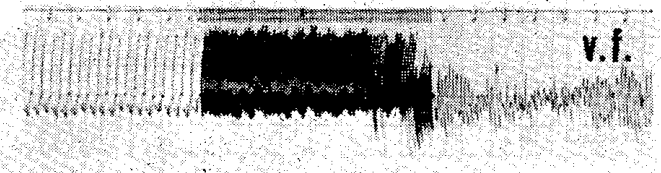

Dog 2183

Fig. 1. A-V block produced by occluding the septal arteries, and restoration of the A-V conduction after releasing the occlusion.

a) Dog No. 165. PR prologation, A-V block with Wenckebach's period and 2:1 A-V block appeared in succession. $1: 1 \mathrm{~A}-\mathrm{V}$ conduction was restored after releasing the occlusion and the PR interval returned to the previous value in four minutes. The upper arrow indicates the occlusion of the septal arteries and the lower one indicates the release of the clamp. Paper seed $50 \mathrm{~mm} / \mathrm{sec}$.

b) Dog No. 218. A ventricular bigeminy appeared about 50 seconds after the occlusion. A-V block followed and progressed from $2: 1$ to $4: 1$, and finally to complete A-V block in 7 minutes. Immediately after releasing the clamps complete $\mathrm{A}-\mathrm{V}$ block was converted to $2: 1$ block and later to $1: 1$ sinus rhythm. A ventricular fibrillation ensued. Two arrows drawn above the first strip indicate the occlusion of the anterior and posterior septal arteries respectively. Two arrows below indicate the release of the clamps. The paper speed was changed several times in the recordings. Interval timer indicates 1 sec.

†(Received for Publication, July 29, 1965) 